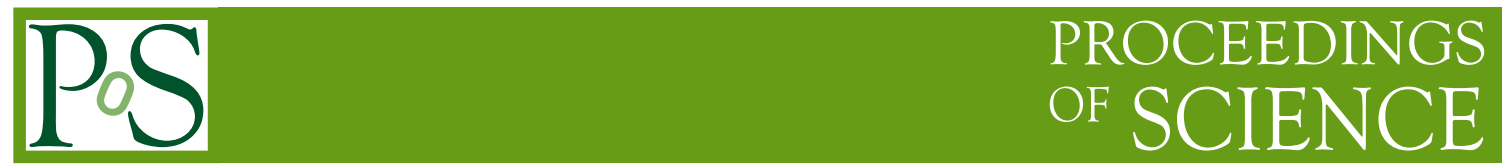

\title{
QCD axion and dark matter
}

\author{
Kiwoon Choi ${ }^{* \dagger}$ \\ Center for Theoretical Physics of the Universe, Institute for Basic Science \\ Daejeon, 34051, Korea \\ E-mail: kchoilibs.re.kr
}

I discuss the axion solution of the strong CP problem and its cosmological implications, while focusing on the prospect for the QCD axion dark matter.

The 3rd International Symposium on "Quest for the Origin of Particles and the Universe"

5-7 January 2017

Nagoya University, Japan

\footnotetext{
* Speaker.

${ }^{\dagger}$ This work was supported by IBS under the project code IBS-R018-D1.
} 


\section{Introduction}

The strong CP problem [1] is a naturalness problem of the Standard Model (SM) of particle physics, associated with the $\mathrm{CP}$ violation in the model. The SM involves two $\mathrm{CP}$ violating angle parameters: (i) the QCD angle $\bar{\theta}=\theta_{\mathrm{QCD}}+\arg \left(y_{u} y_{d}\right)$ which can cause $\mathrm{CP}$ violation in the strong interactions, where $\theta_{\mathrm{QCD}}$ is the bare QCD angle and $y_{u}, y_{d}$ are the $3 \times 3$ Yukawa coupling matrices of the up and down-type quarks, and (ii) the Kobayashi-Maskawa phase $\delta_{\mathrm{KM}}$ which explains the observed CP violation in the weak interactions. Although $\bar{\theta}$ and $\delta_{\mathrm{KM}}$ share the origin from the quark Yukawa couplings, their values are so different. $|\bar{\theta}|$ is required to be smaller than $10^{-10}$ to be consistent with the non-observation of the neutron electric dipole moment, while $\delta_{\mathrm{KM}} \sim 1$ to explain the CP-violating decays of the $K$ and $B$ mesons. To understand this puzzle, many ideas have been proposed so far. Perhaps the simplest solution would be that one of the light quarks, e.g. the up-quark, is massless, rendering $\bar{\theta}$ to be dynamically relaxed to zero by the condensation of the up quark field [2]. However recent lattice calculations suggest that a massless up-quark is inconsistent with low energy hadron data [3]. Another solution, which is still viable, is that $\mathrm{CP}$ is an exact symmetry of the underlying theory, but spontaneously broken in a way to yield $\delta_{\mathrm{KM}} \sim 1$, while keeping $\theta_{\mathrm{QCD}}$ suppressed enough [4] . The third, perhaps the most compelling, solution is to introduce an anomalous global U(1) symmetry, the Peccei-Quinn (PQ) symmetry, which is nonlinearly realized even at high scales above the weak scale [5]. This solution predicts a very weakly coupled light pseudo-Nambu-Goldstone boson, the QCD axion, whose vacuum expectation value, which can be identified as $\bar{\theta}$, is determined to be vanishing by the axion potential generated by the QCD anomaly $[6,7,8]$.

One of the most interesting consequence of the PQ solution of the strong $\mathrm{CP}$ problem is that the predicted axions can constitute the observed dark matter in our universe [9]. In this talk, I discuss some basics features of the QCD axion, while focusing on the cosmological implications and the prospect for axion dark matter.

\section{Axion solution of the strong CP problem}

The PQ solution of the strong CP problem assumes a global $U(1)_{\mathrm{PQ}}$ symmetry which is explicitly broken dominantly by the QCD anomaly. To be consistent with low energy phenomenology, the PQ symmetry should be non-linearly realized in low energy limit, with an axion decay constant $f_{a}$ well above the weak scale. At scales below $f_{a}$, one can always make a proper field redefinition to make only the axion field $a(x)$ transforms under $U(1)_{\mathrm{PQ}}$, while all other fields are invariant [10]:

$$
U(1)_{\mathrm{PQ}}: \quad \frac{a}{f_{a}} \rightarrow \frac{a}{f_{a}}+\text { constant }, \quad \Phi \rightarrow \Phi \quad\left(\frac{a}{f_{a}} \equiv \frac{a}{f_{a}}+2 \pi\right),
$$

where $\Phi$ stands for all light degrees of freedom at scales below $f_{a}$ other than the axion, and $f_{a}$ is defined as the periodicity of the canonically normalized axion field. Generically this non-linear PQ symmetry can be broken explicitly by various forms of non-derivative couplings of the axion field, including the coupling to the QCD anomaly. At leading order in the derivative expansion, generic Wilsonian effective lagrangian of the axion field can be written as

$$
\mathscr{L}_{\text {eff }}=\frac{1}{2} \partial_{\mu} a \partial^{\mu} a+\frac{\partial_{\mu} a}{f_{a}} \tilde{J}^{\mu}+\frac{1}{32 \pi^{2}} \frac{a}{f_{a}} \sum_{i} c_{i} F^{i \mu v} \tilde{F}_{\mu \nu}^{i}+\Delta \mathscr{L},
$$


where

$$
\tilde{J}^{\mu}=\sum_{\psi} c_{\psi} \bar{\psi} \sigma^{\mu} \psi+i \sum_{\phi} c_{\phi}\left(\phi^{\dagger} D^{\mu} \phi-\left(D^{\mu} \phi\right)^{\dagger} \phi\right)
$$

for $\psi$ and $\phi$ denoting the Weyl fermions and complex scalar fields in the model at scales under consideration, $F_{\mu \nu}^{i}(i=3,2,1)$ are the field strength of the SM gauge fields of $S U(3)_{c} \times S U(2)_{W} \times$ $U(1)_{Y}$, and the last term stands for non-derivative axion couplings other than the couplings to $F^{i \mu v} \tilde{F}_{\mu \nu}^{i}$. In our convention, $c_{i}(i=1,2,3)$ are rational numbers, in particular both $c_{3}$ and $c_{2}$ are integer-valued parameters. The Nöether current of the PQ symmetry (2.1) is given by

$$
J_{\mathrm{PQ}}^{\mu}=f_{a} \frac{\delta \mathscr{L}_{\mathrm{eff}}}{\delta \partial_{\mu} a}=f_{a} \partial^{\mu} a+\tilde{J}^{\mu}
$$

with a divergence determined as

$$
\partial_{\mu} J_{\mathrm{PQ}}^{\mu}=f_{a} \frac{\delta \mathscr{L}_{\mathrm{eff}}}{\delta a}=\frac{1}{32 \pi^{2}} \sum_{i} c_{i} F^{i \mu v} \tilde{F}_{\mu v}^{i}+f_{a} \frac{\delta \Delta L_{\mathrm{eff}}}{\delta a} .
$$

With the above explicit breaking of the PQ symmetry, a nonzero axion potential is developed, taking the following form:

$$
V_{\mathrm{axion}}=V_{\mathrm{QCD}}+\Delta V=-f_{\pi}^{2} m_{\pi}^{2} \frac{\sqrt{m_{u}^{2}+m_{d}^{2}+2 m_{u} m_{d} \cos \left(c_{3} a / f_{a}\right)}}{m_{u}+m_{d}}+\Delta V,
$$

where $V_{\mathrm{QCD}}$ is the axion potential induced by the axion coupling to the QCD anomaly $F^{3 \mu \nu} \tilde{F}_{\mu \nu}^{3}$, and $\Delta V$ is the additional potential originating from $\Delta \mathscr{L}$. In the above discussion, we already chose the field basis for which the QCD angle parameter $\bar{\theta}$ can be identified as the vacuum value of $c_{3} a / f_{a}$. One then finds

$$
\bar{\theta} \equiv c_{3}\left\langle\frac{a}{f_{a}}\right\rangle \sim \frac{\Delta V}{f_{\pi}^{2} m_{\pi}^{2}}
$$

for $|\Delta V| \lesssim f_{\pi}^{2} m_{\pi}^{2}$. Obviously one needs two condition to solve the strong CP problem with a PQ symmetry: (i) $c_{3} \neq 0$, i.e. $U(1)_{\mathrm{PQ}}$ should be explicitly broken by the $\mathrm{QCD}$ anomaly, and (ii) $\mathrm{PQ}$ symmetry breaking other than the QCD anomaly, which are encoded in $\triangle \mathscr{L}$, should be negligible enough to yield

$$
|\Delta V| \lesssim 10^{-10} f_{\pi}^{2} m_{\pi}^{2}
$$

As for a more detailed feature of the QCD axion, there can be two distinctive type of models. One is the KSVZ-type model [7] with $c_{\psi}=0$ at tree level for the SM fermions $\psi$, and the other is the DFSZ-type model [8] with $c_{\psi}=\mathscr{O}(1)$. Regardless of this detail, the QCD axion has the following mass and couplings (to the photon and nucleons), which can be characterized by the single parameter $f_{a}$ :

$$
\begin{gathered}
m_{a} \sim 5 \times 10^{-6}\left(10^{12} \mathrm{GeV} / f_{a}\right) \mathrm{eV}, \\
\frac{1}{2} g_{a \gamma \gamma} a \vec{E} \cdot \vec{B} \text { with } g_{a \gamma \gamma} \sim 10^{-15}\left(10^{12} \mathrm{GeV} / f_{a}\right) \mathrm{GeV}^{-1}, \\
g_{a N N} \partial_{\mu} a \bar{N} \gamma^{\mu} \gamma_{5} N \text { with } g_{a N N} \sim 10^{-12}\left(10^{12} \mathrm{GeV} / f_{a}\right) \mathrm{GeV}^{-1} .
\end{gathered}
$$


Among the many constraints on the QCD axion, the most stringent one comes from the astrophysical consideration of the star cooling by axion emission [1], implying

$$
f_{a} \gtrsim 4 \times 10^{8} \mathrm{GeV} .
$$

An immediate consequence of this lower bound on the axion decay constant is that the QCD axion is very light and very weakly coupled, so that its lifetime is much longer than the age of the universe. As a result, once the QCD axions were produced in the early universe, they constitute (part of) the dark matter in the present universe [9].

\section{Production of axion dark matter}

There are two independent mechanisms to produce the QCD axions in the early universe. One is the so-called misalignment mechanism [9], which is based on the observation that the axion mass in the early universe well before the QCD phase transition is negligible compared to the Hubble expansion rate, and therefore generically there can be a misalignment between the initial value of the axion field and the present vacuum value minimizing the axion potential (2.5). The other possible source of axion dark matter is the collapsing network of axionic strings and domain walls [11]. In the pre-inflation scenario that the last PQ phase transition took place before the primordial inflation is over, all axionic strings were inflated away, and therefore there is no network of axionic strings and domain walls within the horizon of visible universe. In such case, axions are produced mostly by the misalignment mechanism. On the other hand, in the post-inflation scenario in which the last PQ phase transition took place after the inflation is over, it turns out that the network of axionic strings and walls provide the dominant source of the axion dark matter.

Let us first consider the axion production by misalignment. Classical evolution of the spatially homogeneous axion field in the early universe is governed by

$$
\ddot{a}+3 H \dot{a}+\frac{\partial V}{\partial a}=0,
$$

where $H$ is the Hubble expansion rate. For simplicity, we take the harmonic approximation for the axion potential:

$$
V \approx \frac{1}{2} m_{a}^{2}(t) a^{2}(t)
$$

where the temperature dependence of the axion mass in the early universe can be approximated as

$$
m_{a}(T) \propto \frac{1}{f_{a}}\left(\frac{\Lambda_{\mathrm{QCD}}}{T}\right)^{n}
$$

with $n=0$ for $T \ll \Lambda_{\mathrm{QCD}}$ and $n \approx 4$ for $T \gg \Lambda_{\mathrm{QCD}}$ for the QCD scale $\Lambda_{\mathrm{QCD}}=\mathscr{O}(100) \mathrm{MeV}$. The corresponding energy and pressure densities of the homogeneous axion field are given by

$$
\rho_{a}=\frac{1}{2}\left(\dot{a}^{2}+m_{a}^{2} a^{2}\right), \quad p_{a}=\frac{1}{2}\left(\dot{a}^{2}-m_{a}^{2} a^{2}\right),
$$

and the axion energy density evolves as

$$
\dot{\rho}_{a}=-3 H \dot{a}^{2}+\dot{m}_{a} m_{a} a^{2} \quad\left(\frac{\dot{m}_{a}}{m_{a}} \approx-n \frac{\dot{T}}{T} \approx n H\right) .
$$


During the early universe well before the QCD phase transition, one has $H(t) \gg m_{a}(t)$, and then the axion field is effectively a constant over the Hubble time scale $\Delta t \sim 1 / H$. On the other hand, after the QCD phase transition, $H \ll m_{a}$ and the axion field enters into the regime of damped oscillation, with the oscillation time scale $\delta t \sim 1 / m_{a}$ shorter than $\Delta t \sim 1 / H$. In this regime, it is convenient to take an average over the oscillation period $\delta t$, and focus on the slow dynamics over the time scale longer than the Hubble time. One then finds

$$
\frac{\left\langle\dot{a}^{2}(t)\right\rangle}{\left\langle m_{a}^{2}(t) a^{2}(t)\right\rangle}=1+\mathscr{O}\left(H^{2} / m_{a}^{2}\right)
$$

where the brackets mean the average over the oscillation period, and we used $\dot{m}_{a} / m_{a} \lesssim \mathscr{O}(H)$ to get the above result. This shows that axions are pressureless up to small corrections of $\mathscr{O}\left(H^{2} / m_{a}^{2}\right)$, i.e. $\left\langle p_{a}\right\rangle=\mathscr{O}\left(H^{2} / m_{a}^{2}\right)$, and the averaged axion energy density $\left\langle\rho_{a}\right\rangle \approx\left\langle\dot{a}^{2}\right\rangle \approx\left\langle m_{a}^{2}(t) a^{2}(t)\right\rangle$ follows the evolution equation:

$$
\frac{d}{d t}\left\langle\rho_{a}\right\rangle=\left(-3 H+\frac{\dot{m}_{a}}{m_{a}}\right)\left\langle\rho_{a}\right\rangle
$$

One then finds that the effective axion number density defined as follow has a simple cosmic evolution:

$$
n_{a} \equiv \frac{\left\langle\rho_{a}\right\rangle(t)}{m_{a}(t)} \propto \frac{1}{R^{3}(t)},
$$

where $R$ is the scale factor of the expanding universe. In other words, during the regime of $H \ll m_{a}$, $n_{a}$ evolves like the number density of pressureless non-relativistic particles.

With the above results, one can estimate the relic axion mass density $\rho_{a}\left(t_{0}\right)$ in the present universe. For this, let us examine the parametric dependence of $\rho_{a}\left(t_{0}\right)$ on $f_{a}$ and the initial misalignment of the axion field. We first note that the axion fields enters into the damped oscillation regime around the time $t_{\text {osc }}$ when

$$
m_{a}\left(t_{\mathrm{osc}}\right) \approx 3 H\left(t_{\mathrm{osc}}\right)
$$

Using $n_{a} \propto 1 / R^{3}$, we find

$$
\frac{\rho_{a}\left(t_{0}\right)}{m_{a}\left(t_{0}\right)} \approx \frac{\rho_{a}\left(t_{\mathrm{osc}}\right)}{m_{a}\left(t_{\mathrm{osc}}\right)}\left(\frac{R\left(t_{\mathrm{osc}}\right)}{R\left(t_{0}\right)}\right)^{3}=\frac{\rho_{a}\left(t_{\mathrm{osc}}\right)}{m_{a}\left(t_{\mathrm{osc}}\right)} \frac{s\left(t_{0}\right)}{s\left(t_{\mathrm{osc}}\right)},
$$

where $s(t)$ denotes the entropy density, and $t_{0}$ is the present time. The axion energy density at $t_{\mathrm{osc}}$ is given by

$$
\rho_{a}\left(t_{\mathrm{osc}}\right) \approx m_{a}^{2}\left(t_{\mathrm{osc}}\right) a_{i}^{2} \equiv m_{a}^{2}\left(t_{\mathrm{osc}}\right) f_{a}^{2} \theta_{\mathrm{mis}}^{2}
$$

where $a_{i}$ denotes the value of the axion field right before the axion enters into the damped oscillation regime at $t \sim t_{\mathrm{osc}}$, and we introduce the misalignment angle $\theta_{\text {mis }} \equiv a_{i} / f_{a}$ for later convenience. From (3.3) and (3.9), one easily finds that the temperature at $t_{\mathrm{osc}}$ has the following dependence on the axion decay constant:

$$
T_{\mathrm{osc}} \propto 1 / f_{a}^{1 /(n+2)} .
$$


From (3.10), one finds also

$$
\rho_{a}\left(t_{0}\right)=m_{a}\left(t_{0}\right) m_{a}\left(t_{\mathrm{osc}}\right) f_{a}^{2} \theta_{\mathrm{mis}}^{2}\left(\frac{s\left(t_{0}\right)}{s\left(t_{\mathrm{osc}}\right)}\right) \sim m_{a}^{2}\left(t_{0}\right) f_{a}^{2}\left(\frac{m_{a}\left(t_{\mathrm{osc}}\right)}{m_{a}\left(t_{0}\right)}\right)\left(\frac{T_{0}}{T_{\mathrm{osc}}}\right)^{3} \theta_{\mathrm{mis}}^{2} \propto \frac{\theta_{\mathrm{mis}}^{2}}{T_{\mathrm{osc}}^{n+3}},(3 .
$$

which results in the following parametric dependence of the relic axion mass density at present:

$$
\rho_{a}\left(t_{0}\right) \propto f_{a}^{(n+3) /(n+2)} \theta_{\text {mis }}^{2} .
$$

For $f_{a} \lesssim \mathscr{O}\left(10^{17}\right) \mathrm{GeV}$, which is the range of the axion decay constant of our interest, we have $T_{\mathrm{osc}}>\Lambda_{\mathrm{QCD}}$, and then one can approximate the temperature-dependent axion mass (3.3) at $t_{\mathrm{osc}}$ with $n \approx 4$. Finally one can do a bit more detailed analysis to fix the numerical coefficient to find the following relic axion mass density at present in the unit of the critical energy density $\rho_{c}=3 M_{P}^{2} H^{2}$ :

$$
\Omega_{a}(\text { misalignment }) \equiv \frac{\rho_{a}\left(t_{0}\right)}{\rho_{c}\left(t_{0}\right)} \sim 0.2\left(\frac{f_{a}}{10^{12} \mathrm{GeV}}\right)^{7 / 6} \theta_{\text {mis }}^{2} .
$$

Whatever its origin is, if there were a fluctuation of the axion field at $t \sim t_{\mathrm{osc}}$, axion dark matter from misalignment generates an isocurvature perturbation in the power spectrum of the cosmic microwave background (CMB) [12]. A naive estimate of the resulting CMB isocurvature perturbation is as follows. First, a simple scaling argument suggests

$$
\left(\frac{\delta T}{T}\right)_{\text {iso }} \sim \frac{\left(\delta \rho_{\mathrm{DM}}\right)_{\text {iso }}}{\rho_{\mathrm{DM}}} \sim \frac{\delta \rho_{a}}{\rho_{\mathrm{DM}}} \text { after the matter domination, }
$$

where $\rho_{\mathrm{DM}}$ denotes the total dark matter mass density. One finds also

$$
\frac{\delta \rho_{a}}{\rho_{\mathrm{DM}}} \sim \frac{\Omega_{a}}{\Omega_{\mathrm{DM}}} \frac{\delta \rho_{a}}{\rho_{a}} \sim \frac{\Omega_{a}}{\Omega_{\mathrm{DM}}} \frac{\delta \theta}{\theta_{\mathrm{mis}}} \sim\left(\frac{\Omega_{a}}{\Omega_{\mathrm{DM}}}\right)^{1 / 2}\left(\frac{f_{a}}{10^{12} \mathrm{GeV}}\right)^{7 / 12} \delta \theta
$$

where $\delta \theta$ denotes the axion fluctuation in angle unit, and we have used (3.15) for the last expression. In the pre-inflation scenario, axion field experiences a de-Sitter quantum fluctuation, yielding

$$
\delta \theta \sim \frac{H\left(t_{\text {inf }}\right)}{2 \pi f_{a}\left(t_{\text {inf }}\right)},
$$

where $H\left(t_{\text {inf }}\right)$ and $f_{a}\left(t_{\text {inf }}\right)$ denote the Hubble expansion rate and the axion decay constant, respectively, during the inflationary period. Note that, depending upon the mechanism to determine the axion scale [13], $f_{a}\left(t_{\text {inf }}\right)$ can be very different from the present axion decay constant $f_{a}$ [14]. Even when one makes a more detailed analysis [12], it turns out that the resulting CMB isocurvature perturbation is quite close to the above naive estimate, yielding

$$
\left(\frac{\delta T}{T}\right)_{\text {iso }} \sim\left(\frac{\Omega_{a}}{\Omega_{\mathrm{DM}}}\right)^{1 / 2}\left(\frac{f_{a}}{10^{12} \mathrm{GeV}}\right)^{7 / 12}\left(\frac{H\left(t_{\mathrm{inf}}\right)}{2 \pi f_{a}\left(t_{\mathrm{inf}}\right)}\right)<10^{-5},
$$

where we have imposed the recent PLANCK bound on the CMB isocurvature fluctuation [15].

In the post-inflation scenario, axionic strings with a tension $\mu_{\text {string }} \sim f_{a}^{2}$ are produced during the PQ phase transition, which can give rise to an important cosmological consequence. Those strings eventually form a network of strings and domain walls, in which each string is attached by $N_{\mathrm{DW}}$ 
domain walls with a tension $\sigma_{\text {wall }} \sim m_{a} f_{a}^{2}$, which are formed during the QCD phase transition. The number of attached domain walls are determined to be the integer-valued model parameter $c_{3}$, i.e. $N_{\mathrm{DW}}=c_{3}$, which was defined in the axion effective lagrangian (2.2) and the PQ current divergence (2.4). One then finds that axionic strings and domain walls collapse efficiently, while radiating axions, only when $N_{\mathrm{DW}}=1$. In the other case with $N_{\mathrm{DW}} \geq 2$, the network of axionic strings and domain walls cause a disastrous domain wall problem, and therefore in the post-inflation scenario, the axion model should have $N_{\mathrm{DW}}=c_{3}=1$ in order to be cosmologically viable.

To determine the density of relic axions produced by axionic strings and domain walls, one needs to examine the complicate evolution of axionic strings and domain walls [11]. Yet, one can notice without such analysis that the resulting axion mass density has the same dependence on $f_{a}$ as the axion mass density from misalignment, i.e.

$$
\Omega(\text { strings } / \text { walls }) \propto f_{a}^{(n+3) /(n+2)} .
$$

This is because axions are produced dominantly around the time $t_{\text {coll }}$ when the network of strings and domain walls begin to collapse, which took place soon after the Hubble expansion rate becomes comparable to the axion mass:

$$
H\left(t_{\text {coll }}\right) \sim m_{a}\left(t_{\text {coll }}\right),
$$

and the produced axions are nearly non-relativistic as their momenta are comparable to $H$. Around $t_{\text {coll }}$, axionic strings and walls follow a simple scaling behavior, yielding

$$
\begin{aligned}
& \rho_{\text {string }}\left(t_{\text {coll }}\right) \sim \mu_{\text {string }} H^{2}\left(t_{\text {coll }}\right) \sim m_{a}^{2}\left(t_{\text {coll }}\right) f_{a}^{2}, \\
& \rho_{\text {wall }}\left(t_{\text {coll }}\right) \sim \sigma_{\text {wall }} H\left(t_{\text {coll }}\right) \sim m_{a}^{2}\left(t_{\text {coll }}\right) f_{a}^{2} .
\end{aligned}
$$

The resulting axion number density at $t \sim t_{\text {coll }}$ is roughly given by

$$
n_{a}\left(t_{\text {coll }}\right) \sim H\left(t_{\text {coll }}\right) \times\left(\frac{\rho_{\text {string }}+\rho_{\text {wall }}}{\omega_{a}}\right) \sim m_{a}\left(t_{\text {tall }}\right) f_{a}^{2},
$$

where $\omega_{a} \sim H\left(t_{\text {coll }}\right) \sim m_{a}\left(t_{\text {coll }}\right)$ is the typical energy of the axions produced by strings and walls at $t \sim t_{\text {coll }}$. As the produced axions are mostly non-relativistic at $t>t_{\text {coll }}, n_{a}$ evolves as $R^{-3}$. Again this leads to

$$
\rho_{a}\left(t_{0}\right)=m_{a}\left(t_{0}\right) n_{a}\left(t_{0}\right) \sim m_{a}\left(t_{\mathrm{coll}}\right) n_{a}\left(t_{\mathrm{coll}}\right)\left(\frac{R\left(t_{\mathrm{coll}}\right)}{R\left(t_{0}\right)}\right)^{3} \sim m_{a}^{2}\left(t_{0}\right) f_{a}^{2}\left(\frac{m_{a}\left(t_{\mathrm{coll}}\right)}{m_{a}\left(t_{0}\right)}\right)\left(\frac{T_{0}}{T_{\mathrm{coll}}}\right)^{3}
$$

and therefore

$$
\Omega_{a}(\text { string/wall }) \propto f_{a}^{(n+3) /(n+2)} .
$$

The corresponding coefficient can be fixed by numerical simulation for the evolution of axionic strings and domain walls [11], yielding

$$
\Omega_{a}(\text { string } / \text { wall }) \sim(1-5) \times\left(\frac{f_{a}}{10^{12} \mathrm{GeV}}\right)^{7 / 6} .
$$




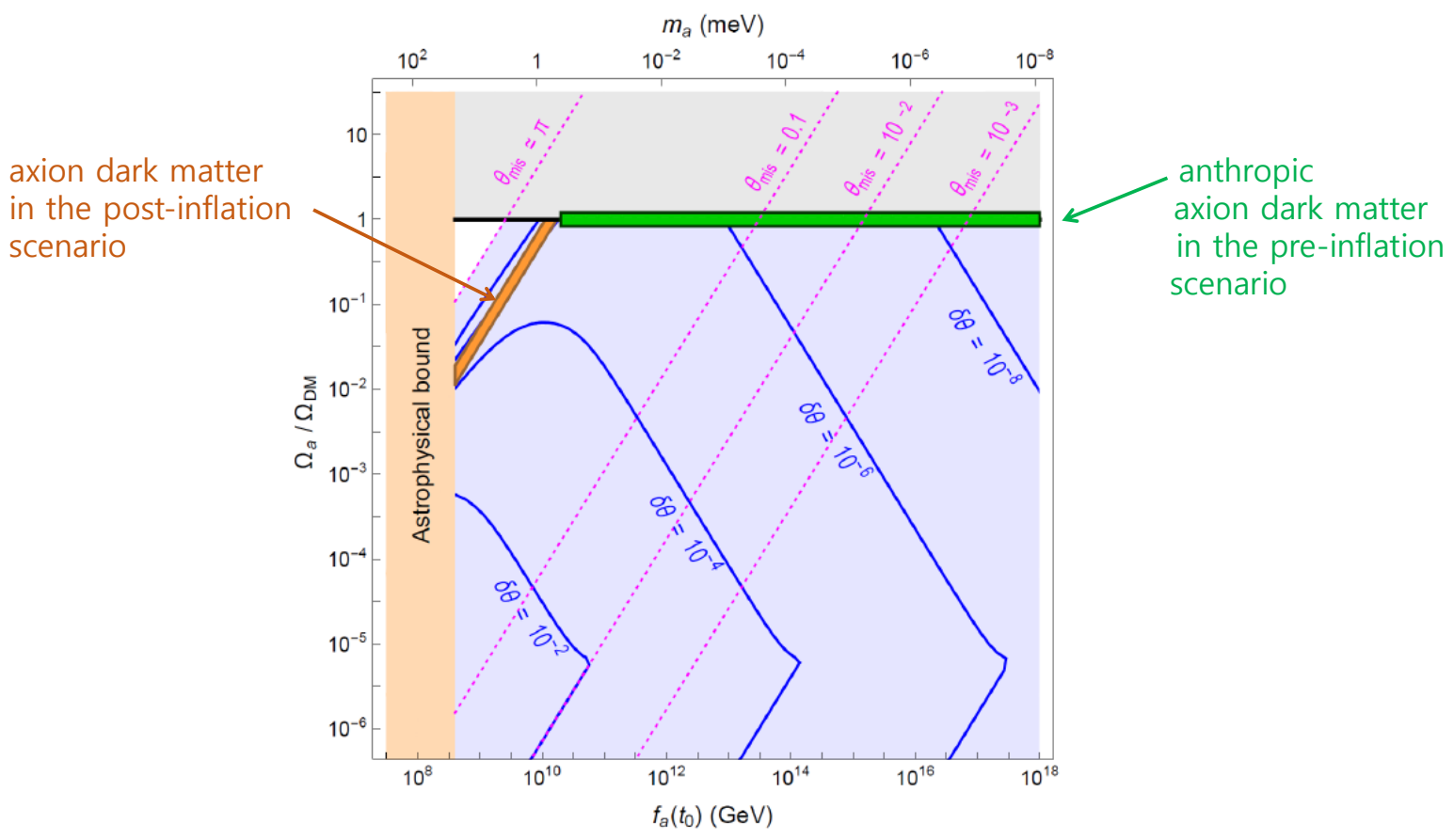

Figure 1: The thin orange band denotes the relic axion mass density in the post-inflation scenario, while the green band represents the case that axions constitute the observed dark matter in the pre-inflation scenario, with a misalignment angle adjusted by the anthropic selection. The blue lines correspond to the upper bound on the axion mass density in the pre-inflation scenario for a given value of $\delta \theta=H\left(t_{\text {inf }}\right) / 2 \pi f_{a}\left(t_{\text {inf }}\right)$.

\section{Conclusion}

The PQ solution of the strong CP problem is particularly attractive as it provides also a compelling candidate for dark matter in the universe. In the pre-inflation scenario in which the last PQ phase transition took place before the primordial inflation is over, dark matter axions are produced mostly by the misalignment mechanism, with a relic density determined by the two parameters, the axion decay constant $f_{a}$ at present and the initial misalignment angle $\theta_{\text {mis }}$. Dark matter axions in this scenario can generate a CMB isocurvature perturbation, whose amplitude is determined by the axion fraction of the dark matter, i.e. $\Omega_{a} / \Omega_{\mathrm{DM}}$, and the inflationary angle fluctuation $\delta \theta=H\left(t_{\text {inf }}\right) / 2 \pi f_{a}\left(t_{\text {inf }}\right)$ which is determined by the inflationary Hubble scale $H\left(t_{\text {inf }}\right)$ and the axion decay constant $f_{a}\left(t_{\text {inf }}\right)$ during the inflationary epoch. On the other hand, in the post-inflation scenario in which the PQ phase transition took place after the inflation, dark matter axions are produced dominantly by the axionic strings and domain walls, which results in the relic density determined by $f_{a}$ alone. To avoid the cosmological domain wall problem, the post-inflation scenario requires the model parameter $c_{3}=1$. In Fig. 1, we summarize the relic density of the QCD axions in both the pre- and post-inflation scenarios, including the constraint from the isocurvature perturbation of CMB [16]. 


\section{References}

[1] For a recent review, see J. E. Kim and G. Carosi, Rev. Mod. Phys. 82, 557 (2010) [arXiv:0807.3125 [hep-ph]].

[2] H. Georgi and I. N. McArthur, HUTP-81/A011; D. B. Kaplan and A. V. Manohar, Phys. Rev. Lett. 56, 2004 (1986); K. Choi, C. W. Kim and W. K. Sze, Phys. Rev. Lett. 61, 794 (1988).

[3] For a recent review, see S. Aoki et al., Eur. Phys. J. C 77, no. 2, 112 (2017) [arXiv:1607.00299 [hep-lat]].

[4] A. E. Nelson, Phys. Lett. 136B, 387 (1984); S. M. Barr, Phys. Rev. Lett. 53, 329 (1984).

[5] R. D. Peccei and H. R. Quinn, Phys. Rev. Lett. 38, 1440 (1977).

[6] S. Weinberg, Phys. Rev. Lett. 40, 223 (1978); F. Wilczek, Phys. Rev. Lett. 40, 279 (1978).

[7] J. E. Kim, Phys. Rev. Lett. 43, 103 (1979); M. A. Shifman, A. I. Vainshtein and V. I. Zakharov, Nucl. Phys. B 166, 493 (1980).

[8] M. Dine, W. Fischler and M. Srednicki, Phys. Lett. 104B, 199 (1981); A. R. Zhitnitsky, Sov. J. Nucl. Phys. 31, 260 (1980) [Yad. Fiz. 31, 497 (1980)].

[9] J. Preskill, M. B. Wise and F. Wilczek, Phys. Lett. 120B, 127 (1983); L. F. Abbott and P. Sikivie, Phys. Lett. 120B, 133 (1983); M. Dine and W. Fischler, Phys. Lett. 120B, 137 (1983).

[10] H. Georgi, D. B. Kaplan and L. Randall, Phys. Lett. 169B, 73 (1986).

[11] For a recent discussion of this issue, see M. Kawasaki, K. Saikawa and T. Sekiguchi, Phys. Rev. D 91, no. 6, 065014 (2015) [arXiv:1412.0789 [hep-ph]].

[12] For a more detailed discussion of this issue, see P. Fox, A. Pierce and S. D. Thomas, hep-th/0409059.

[13] K. Choi, E. J. Chun and J. E. Kim, Phys. Lett. B 403, 209 (1997) [hep-ph/9608222].

[14] A. D. Linde, Phys. Lett. B 259, 38 (1991).

[15] PLANCK collaboration, P.A.R. Ade et al., arXiv:1303.5082.

[16] K. Choi, K. S. Jeong and M. S. Seo, JHEP 1407, 092 (2014) [arXiv:1404.3880 [hep-th]]. 